\title{
Genetic Association of Recovery from Eating Disorders: The Role of GABA Receptor SNPs
}

\begin{abstract}
Cinnamon S Bloss', Wade Berrettini ${ }^{2}$, Andrew W Bergen ${ }^{3}$, Pierre Magistretti ${ }^{4}$, Vikas Duvvuri ${ }^{5}$, Michael Strober ${ }^{6}$, Harry Brandt ${ }^{7}$, Steve Crawford ${ }^{7}$, Scott Crow $^{8}$, Manfred M Fichter ${ }^{9}$, Katherine A Halmi ${ }^{10}$, Craig Johnson' ', Allan S Kaplan '2,13,14, Pamela Keel' ${ }^{15}$, Kelly L Klump ${ }^{16}$, James MitchelI 17,18, Janet Treasure ${ }^{19}$, D Blake Woodside ${ }^{13,14}$, Enrica Marzola ${ }^{1,20}$, Nicholas J Schork ${ }^{1,21}$ and Walter H Kaye*,5
\end{abstract}

'Scripps Genomic Medicine, Scripps Translational Science Institute, and Scripps Health, La Jolla, CA, USA; ${ }^{2}$ Department of Psychiatry, University of Pennsylvania, Philadelphia, PA, USA; ${ }^{3}$ Center for Health Sciences, SRI International, Menlo Park, CA, USA; ${ }^{4}$ Brain Mind Institute, EPFL and Department of Psychiatry, University of Lausanne Medical School, Lausanne, Switzerland; ${ }^{5}$ Department of Psychiatry, University of California, San Diego, CA, USA; ${ }^{6}$ Department of Psychiatry and Biobehavioral Sciences, David Geffen School of Medicine, University of Califormia at Los Angeles, Los Angeles, CA, USA; 'Department of Psychiatry, University of Maryland School of Medicine, Baltimore, MD, USA; ${ }^{8}$ Department of Psychiatry, University of Minnesota, Minneapolis, MN, USA; ${ }^{9}$ Roseneck Hospital for Behavioral Medicine, Prien, Germany and Department of Psychiatry, University of Munich (LMU), Munich, Germany; ${ }^{10}$ New York Presbyterian Hospital-Westchester Division, Weill Medical College of Cornell University, White Plains, NY, USA; "'Laureate Psychiatric Clinic and Hospital, Tulsa, OK, USA; ${ }^{2}$ Centre for Addiction and Mental Health, Toronto, ON, Canada; ${ }^{13}$ Department of Psychiatry, University of Toronto, Toronto, ON, Canada; ${ }^{14}$ Department of Psychiatry, Toronto General Hospital, University Health Network, Toronto, ON, Canada; ${ }^{15}$ Department of Psychology, Florida State University, Tallahasseei, FL, USA; ${ }^{16}$ Department of Psychology, Michigan State University, East Lansing, MI, USA; ${ }^{17}$ Neuropsychiatric Research Institute, Fargo, ND, USA; ${ }^{18}$ Department of Clinical Neuroscience, University of North Dakota School of Medicine and Health Sciences, Grand Forks, ND, USA; ${ }^{19}$ Eating Disorders Section, Institute of Psychiatry, King's College, University of London, London, UK; ${ }^{20}$ Day Hospital of the Eating Disorders Program at the San Giovanni Battista Hospital of Turin, Turin, Italy; ${ }^{2}$ 'Department of Molecular and Experimental Medicine, The Scripps Research Institute, La Jolla, CA, USA

Follow-up studies of eating disorders (EDs) suggest outcomes ranging from recovery to chronic illness or death, but predictors of outcome have not been consistently identified. We tested 5151 single-nucleotide polymorphisms (SNPs) in approximately 350 candidate genes for association with recovery from ED in 1878 women. Initial analyses focused on a strictly defined discovery cohort of women who were over age 25 years, carried a lifetime diagnosis of an ED, and for whom data were available regarding the presence ( $n=36$ I ongoing symptoms in the past year, ie, 'ill') or absence ( $n=115$ no symptoms in the past year, ie, 'recovered') of ED symptoms. An intronic SNP ( $\mathrm{r} \mid 753621 \mathrm{I})$ in GABRG I showed the strongest statistical evidence of association $\left(p=4.63 \times 10^{-6}\right.$, false discovery rate $(F D R)=0.021$, odds ratio $(O R)=0.46)$. We replicated these findings in a more liberally defined cohort of women age 25 years or younger ( $n=464$ ill, $n=107$ recovered; $p=0.0336, O R=0.68$; combined sample $p=4.57 \times 10^{-6}, F D R=0.0049, O R=0.55$ ). Enrichment analyses revealed that GABA ( $\gamma$-aminobutyric acid) SNPs were over-represented among SNPs associated at $p<0.05$ in both the discovery $(Z=3.64, p=0.0003)$ and combined cohorts $(Z=2.07, p=0.0388)$. In follow-up phenomic association analyses with a third independent cohort ( $n=154 \mathrm{ED}$ cases, $n=677$ controls), rs I75362 II was associated with trait anxiety $(p=0.049)$, suggesting a possible mechanism through which this variant may influence ED outcome. These findings could provide new insights into the development of more effective interventions for the most treatment-resistant patients.

Neuropsychopharmacology (20I I) 36, 2222-2232; doi: I0.1038/npp.20 I I.108; published online I3 July 201 I

Keywords: GABA; anorexia nervosa; recovery from eating disorders; genetic association; single nucleotide polymorphisms; eating/metabolic disorders

*Correspondence: Dr WH Kaye, Department of Psychiatry, University of California, San Diego, 8950 Villa La Jolla Drive, Suite C207, La Jolla, CA 92037, USA, Tel: + I 858205 7293, Fax: + I 8585346727 ,

E-mail: wkaye@ucsd.edu or NJ Schork, Scripps Genomic Medicine, Scripps Translational Science Institute, 3344 North Torrey Pines Court, Suite 300, La Jolla, CA 92037, USA, Tel: + I 858554 5705,

E-mail: nschork@scripps.edu

Received 29 October 2010; revised I May 201 I; accepted 12 May 2011

\section{INTRODUCTION}

The eating disorders (ED), anorexia nervosa (AN) and bulimia nervosa $(\mathrm{BN})$, are serious and complex psychiatric disorders. AN is characterized by an inability to maintain normal body weight and a relentless pursuit of thinness, whereas $\mathrm{BN}$ is characterized by recurrent episodes of binge eating in combination with inappropriate compensatory behavior (American Psychiatric Association, 2000). It has 
been shown that transitions between the two syndromes occur in many cases, and thus it has been argued that AN and BN likely share some risk and liability factors (Lilenfeld et al, 1998; Walters and Kendler, 1995).

Recent studies suggest that premorbid, genetically determined temperament and personality traits contribute to a vulnerability to $\mathrm{AN}$ and $\mathrm{BN}$ during adolescence in women (Kaye et al, 2009; Lilenfeld et al, 2006). Once an ED develops, there is evidence that state alterations, which occur secondarily to pathological eating and/or malnutrition, may sustain the illness and perhaps accelerate a worsening of symptoms in some patients. Individuals with EDs, in particular $\mathrm{AN}$, are often resistant to treatment and lack insight regarding the medical consequences of the disorder. Identification of effective treatments that reverse either or both the trait and state symptoms of AN and $\mathrm{BN}$ has been difficult. Indeed, there are no proven treatments for $\mathrm{AN}$, and while medication and psychotherapies diminish symptoms in $\mathrm{BN}$, most individuals remain symptomatic (Bulik et al, 2007; Shapiro et al, 2007; Walsh, 1991). Although conventional treatments may counteract the worsening of symptoms and minimize medical complications, it remains uncertain whether such treatments increase the number of individuals who have a good longterm outcome. Further, little is known about prognostic factors that identify good or poor outcome (Keel and Brown 2010).

It is well known that individuals with EDs have relatively homogeneous patterns of onset. Specifically, onsets tend to follow a common pattern in that they usually occur in adolescence or young adulthood (ie, typically around the age of puberty), and most cases are women. Now, several reviews (Steinhausen and Weber 2009; Steinhausen 2002) support the contention that course and outcome may also show certain age-dependent patterns. That is, after being ill for 5-10 years, a substantial number of AN (Steinhausen 2002) and BN patients (Keel et al, 1999) show remission of pathological eating and stabilization of weight in their early 20s, although premorbid personality and temperament traits have been shown to persist (Wagner et al, 2006a). Thus, about half of AN and BN patients show full recovery and perhaps a quarter improve considerably. The remaining quarter, however, have a chronic, protracted course, often despite prolonged and repeated episodes of treatment. Moreover, mortality rates are known to be high. One review estimated the aggregate mortality rate of $\mathrm{AN}$ at $0.56 \%$ per year, or approximately $5.6 \%$ per decade (Sullivan 1995), and a more recent study (Crow et al, 2009) found that crude mortality rates over $8-25$ years were $4.0 \%$ for $\mathrm{AN}, 3.9 \%$ for $\mathrm{BN}$, and $5.2 \%$ for EDs not otherwise specified (ED-NOS), which is a diagnostic category used when a person has some symptoms of disordered eating, but does not meet full criteria for $\mathrm{AN}$ or $\mathrm{BN}$.

The substantial fraction of patients who remain ill, coupled with the high mortality rates associated with these disorders, leads one to ask questions about factors that may influence recovery, including both spontaneous as well as in response to treatment. Do some individuals suffer scars related to malnutrition or other factors during the ill state that interfere with recovery? Or, alternatively, are there predisposing genetic factors that contribute to good $v s$ poor outcome? Given the substantial genetic component in the etiology of EDs in general, it follows that there may be genetic variants that contribute to the likelihood of recovery. Identification of such variants may lead to more personalized treatment protocols that include more effective psychotherapies and/or pharmacological interventions for the most treatment-resistant patients. As such, within the context of a large-scale candidate gene study originally designed to evaluate genes that may have a role in the genetic susceptibility to EDs (Pinheiro et al, 2010), we tested genetic associations with recovery from EDs as a primary phenotype. Utilizing a tiered design, we initially performed these analyses in a strictly defined discovery cohort of women who were over age 25 years, carried a lifetime diagnosis of $\mathrm{AN}, \mathrm{BN}$, or ED-NOS, and for whom data were available regarding the presence or absence of ED symptoms in the past year. We then sought to replicate our findings in a more liberally defined cohort of women age 25 years or younger. Finally, we followed up on findings from these primary association studies with secondary phenomic association analyses with a third independent cohort of women to identify possible mechanisms through which our most strongly associated single-nucleotide polymorphism (SNP) may influence outcome.

\section{MATERIALS AND METHODS}

\section{Participant Selection}

Participants were selected from among those enrolled in the three multisite, International Price Foundation (PF) Genetic Studies of Eating disorders, which include the Anorexia Nervosa Affected Relative Pair Study, the Bulimia Nervosa Affected Relative Pair Study, and the Anorexia Nervosa Trios Study. Detailed descriptions of each study, including the criteria for inclusion and exclusion, have been published elsewhere (Kaye et al, 2000, 2004b; Pinheiro et al, 2010; Reba et al, 2005). Each study was designed to identify susceptibility loci involved in risk for EDs. All sites received approval from their local Institutional Review Board, and informed consent was obtained from all study participants.

From the three studies above, a total of 2257 individuals were selected for inclusion in the primary candidate gene association study (Pinheiro et al, 2010). A diagnostic hierarchy was applied for further selection with greater weight (from highest to lowest) placed on inclusion of restricting AN, AN with purging but no binge eating, AN with binge eating with or without purging, a lifetime history of both $\mathrm{AN}$ and BN (ANBN), subthreshold AN with no binge eating or purging, purging $\mathrm{BN}$, and subthreshold $\mathrm{BN}$. The individual from each family with the diagnosis that was highest on the hierarchy was selected; these participants, as well as all 677 control women from the AN Trios Study, were selected as the primary samples for inclusion. A secondary set of samples, which was made up of individuals who were related to one individual in the primary sample, was also selected based on the above criteria.

Genomic DNA samples from the individuals identified above were sent to Illumina for genotyping. Of these, we removed 11 due to genotyping failure, 220 who were originally included for quality control (QC) purposes, 27 male participants with AN, and 121 first-degree relatives. No participants were excluded for excessive genotype 
Table I Sample Characteristics

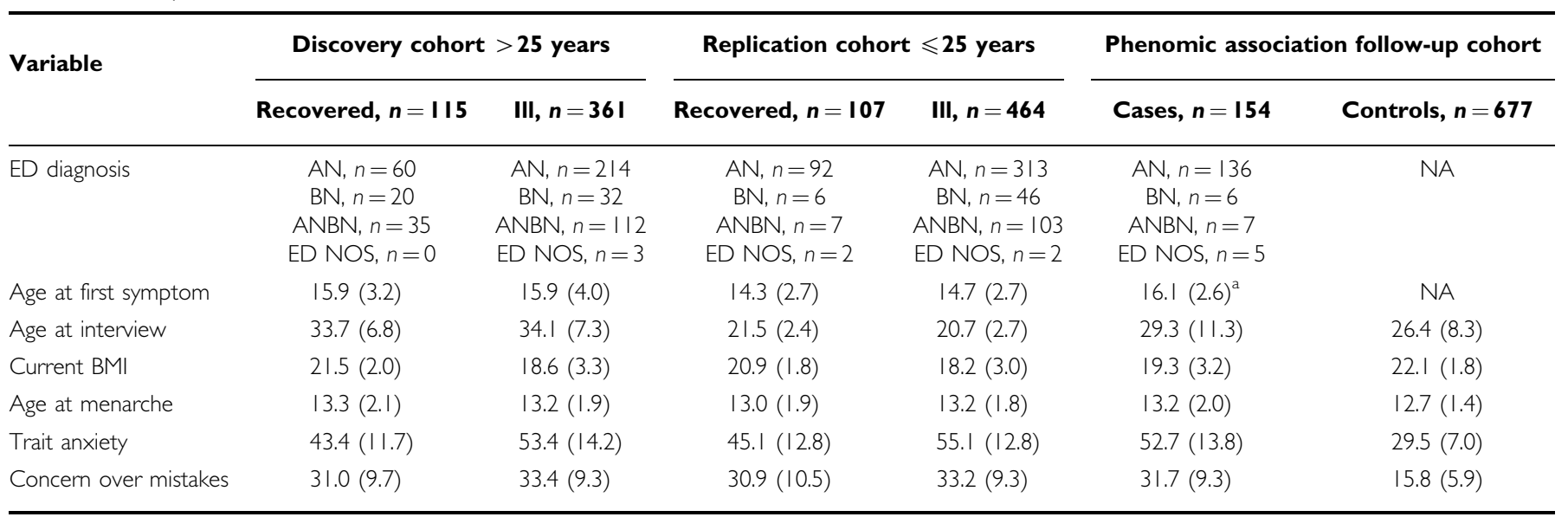

NA $=$ not applicable.

With the exception of ED diagnosis, all values are presented as mean (SD).

${ }^{a}$ For the variable age at first symptom, data were only available for $n=8$ individuals in this group.

missingness (all were $>0.021$ ). As such, the final sample for this study includes 1878 female participants (1201 cases and 677 controls). Additional details on candidate gene study sample selection and QC procedures are provided elsewhere (Pinheiro et al, 2010).

\section{Definition of Discovery, Replication, and Follow-Up Cohorts for Outcome Study}

Recovery was defined as at least 1 year without any ED symptoms (ie, low weight, dieting, binge eating, or compensatory behaviors). These criteria were assessed in the context of the Structured Clinical Interview for DSM-IV Axis I Disorders (First et al, 1997). In our initial test of genetic association with ED outcome, we utilized a strictly defined subset of our sample termed the 'discovery cohort'. These were women who were over age 25 years, carried a lifetime diagnosis of either AN, BN, or ED-NOS (ie, subthreshold AN or BN), and for whom data were available regarding the presence $(n=361$ endorsed ongoing ED symptoms in the past year and considered (ill') or absence ( $n=115$ no ED symptoms in the past year and considered 'recovered') of ED symptoms. Although our definition of recovery in terms of time frame (ie, 1 year without symptoms) is less conservative than what has been used in some previous studies of outcome (Von Holle et al, 2008), there is evidence that a symptom-free period at an older age (eg, mid-20s) is more likely to represent a true recovery compared with younger ages when relapse is more apt to occur (Keel et al, 1999; Steinhausen, 2002; Wagner et al, 2006b). Thus, in restricting our discovery cohort to individuals over age 25 years, we felt that our definition of recovery was reasonably conservative.

To follow-up on our initial findings, we identified a more liberally defined 'replication cohort' of women who were classified according to the above criteria, but who were age 25 years or younger ( $n=464$ ill, $n=107$ recovered). Having observed evidence of replication (see Results below), we identified a third independent 'follow-up cohort' $(n=154$ ED cases, recovery status was unknown, and $n=677$ controls) to conduct secondary phenomic association analysis of the SNP for which we observed the strongest statistical evidence of association with outcome in our discovery and replication cohorts. Table 1 presents descriptive statistics and the breakdown of diagnostic categories represented in each of our three cohorts.

\section{Traits Tested in Phenomic Association Analysis}

As described above, we conducted secondary phenomic association of our top SNP. It is known that carefully selected phenotypes are important for optimizing findings regarding genetic effects in psychiatric disorders (Devlin et al, 2002; Grice et al, 2002). Thus, in terms of selection of phenotypes to test for association, we relied on past work in this area. Specifically, phenotypes have been previously identified for linkage studies in EDs based on the following: evidence of (1) consistent association with eating pathology; (2) heritability; (3) and the extent to which the phenotype reflects trait-like qualities $v s$ state-like qualities that result from the illness (Bulik et al, 2005). On the basis of these criteria, we chose age at menarche in addition to anxiety and perfectionism. Anxiety was indexed by the Trait Anxiety Scale of the Spielberger State-Trait Anxiety Inventory Form Y (Spielberger et al, 1983), and perfectionism was measured by the Concern Over Mistakes subscale from The Multidimensional Perfectionism Scale (MPS) (Frost et al, 1990). We selected this subscale, rather than a total score from the MPS, because it has been previously shown to be uniquely associated with EDs (Bulik et al, 2003).

\section{Candidate Gene Selection, SNP Selection, and QC}

Candidate genes were selected by the PF investigators by a consensus process, and this procedure has been described previously (Pinheiro et al, 2010). Briefly, genes were selected based on their proximity to known ED linkage peaks, previous findings reported in the ED literature, published findings in other related disorders, and based on 
participation in biological pathways thought to be implicated in AN. This list was then narrowed by selecting genes with evidence of brain gene expression, genes shown to be estrogen responsive in microarray studies, and consensus among PF investigators to match the available genotyping budget. Because the onset of EDs occurs predominately in female subjects near time to puberty, it has been suspected that some vulnerability to female gonadal steroids may occur. It is for this reason that estrogen responsiveness was chosen as a criterion for gene selection (eg, see Versini et al (2010)).

On the basis of the RefSeq database (Pruitt et al, 2007) accessed in March 2007, this approach yielded 182 candidate genes, which were processed using TAMAL version 2.0 (Hemminger et al, 2006). A full list of these genes is available as supporting material associated with the paper published by Pinheiro et al (2010). SNPs were selected to tag common variation in Europeans, and selection was enriched to include SNPs in splice sites, exons, highly conserved regions, predicted promoter and regulatory regions, and transcription factor binding and microRNA target sites. A final list of 6568 SNPs was sent to Illumina for genotyping with their Custom Infinium Genotyping Beadchips platform. Although these SNPs received favorable design scores, some SNPs failed genotyping; other SNPs were eliminated based on downstream QC filters, which included minor allele frequency $<0.01, \geqslant 2$ Mendel errors, duplicate samples with $\geqslant 2$ disagreements, SNP missingness $>0.05$, differential genotype missingness in cases $v s$ controls at $p<0.01$, and Hardy-Weinberg equilibrium exact at $p<0.01$ in controls. The total number of SNPs to pass these filters was 5151 .

In this study, to address issues of population stratification, all association $p$-values reported are genomic control (GC)-corrected (Devlin and Roeder, 1999). Further, we observed genomic inflation factors of $\lambda=1.011$ for our discovery cohort and $\lambda=1.065$ for our replication cohort, both of which are consistent with an acceptably small amount of population stratification.

\section{Statistical Analysis}

PLINK version 1.07 (Purcell et al, 2007) was used to conduct all genetic association analyses. The standard contingency table test of association comparing allele frequencies between ill and recovered individuals (ie, cases and controls) was the primary test used. To control for multiple comparisons in the analysis of our discovery cohort, we used a false discovery rate (FDR) approach (Benjamini and Hochberg, 1995; Benjamini et al, 2001). In the analysis of our replication cohort, we considered an $\alpha$-level of $p<0.05$ as evidence for replication. In an attempt to assess the technical validity of the signal from our most strongly associated SNP in our discovery cohort, we closely evaluated the strength of the association of flanking SNPs that showed some degree of linkage disequilibrium (LD) with our top SNP. Haplotype analyses using a 3-SNP sliding window approach were also conducted. Finally, logistic regression was used for additional analyses conditioning on our most strongly associated (or 'top'-ranked) SNP, as well as testing for SNP $\times$ SNP interactions with our top SNP.
Given that this was a large-scale candidate gene study designed based on a priori hypotheses regarding the genes, pathways, and biological systems involved in susceptibility to EDs, we evaluated our top SNP with respect to the networks of genes and pathways in which it participates. Specifically, we conducted new annotation of all of the SNPs in our data set using WGAViewer version 1.26G (Ge et al, 2008), accessed February 2010. Owing to the ever-evolving nature of human genome annotation, this updated annotation revealed that the 5151 SNPs in our data set mapped to over 350 genes; we note, however, that the coverage of genetic variation on these genes was likely less complete. Our top hit fell within a GABA-A ( $\gamma$-aminobutyric acid-A) subunit receptor gene, and thus we evaluated the extent to which SNPs in GABA genes, overall, were enriched or overrepresented among SNPs associated with ED outcome at $p<0.05$. Specifically, we compared the proportion of associated SNPs in GABA genes to the proportion of associated SNPs in non-GABA genes using a $Z$-test.

Finally, SPSS 14.0 was used to conduct follow-up phenomic association analysis of our top SNP with three quantitative traits as described above. Association was tested by separate linear regression analyses with the three primary phenotypes of interest each serving as dependent variables. Given that this analysis was performed with a third independent cohort that consisted of ED cases at varying stages in the course of illness, as well as control women, all models included both diagnosis (dummy-coded $0=$ control or $1=\mathrm{ED}$ ) and current body mass index (BMI) as covariates. The SNP effect was modeled additively as dosage of the minor allele.

\section{RESULTS}

\section{Sample Characteristics}

As shown in Table 1, the majority of ED cases included in the study are individuals with a lifetime diagnosis of either AN or both AN and BN (ANBN). Further, consistent with the literature and as expected, across cohorts, ill individuals show lower current BMI, higher trait anxiety, and higher concern over mistakes. A similar pattern is seen when comparing cases to controls, and control women also show lower age at menarche relative to cases. In addition, the fact that age at interview is higher in the discovery cohort is also to be expected given that this group of individuals was chosen based on their older age (ie, $>25$ years).

\section{Primary Genetic Association Analysis}

Table 2 presents the 25 most statistically significant SNPs from the genetic association analysis of outcome in our discovery cohort. In addition, for each of these SNPs we list the $p$-value observed in both the replication and combined samples, respectively. As shown, rs17536211, an intronic SNP in GABRG1 on chromosome 4, showed the strongest statistical evidence of association with a GC-corrected $p$-value of $4.63 \times 10^{-6}$, which corresponds to an FDR of 0.021 (Figure 1). The odds ratio (OR) observed for this SNP is 0.46 , suggesting that possession of copies of the minor allele is protective from long-term chronic illness (ie, it is associated with recovery). Figure 2 shows the Q-Q plot for 
Table 2 Results of Primary Genetic Association Analysis

\begin{tabular}{|c|c|c|c|c|c|c|c|c|c|c|c|}
\hline SNP & Chr & Position & $\begin{array}{l}\text { MA freq } \\
\text { rec }\end{array}$ & $\begin{array}{c}\text { MA } \\
\text { freq ill }\end{array}$ & SNP type & Gene & $\begin{array}{l}\text { Disc GC } \\
p \text {-value }\end{array}$ & $\begin{array}{l}\text { Disc } \\
\text { OR }\end{array}$ & $\begin{array}{c}\text { Repl GC } \\
\text { p-value }\end{array}$ & $\begin{array}{c}\text { Comb GC } \\
p \text {-value }\end{array}$ & $\begin{array}{c}\text { Comb } \\
\text { OR }\end{array}$ \\
\hline rs|753621। & 4 & 46087733 & 0.33 & 0.18 & INTRONIC & GABRGI & 4.63E-06 & 0.46 & 0.03337 & 4.57E-06 & 0.55 \\
\hline rs4425006 & 19 & 10813364 & 0.13 & 0.06 & INTRONIC & QTRTI & 0.000168 & 0.39 & 0.6345 & 0.03383 & 0.66 \\
\hline rs 16467 & 7 & 24342125 & 0.06 & 0.02 & INTERGENIC & NPY & 0.001443 & 0.32 & 0.5386 & 0.1639 & 0.69 \\
\hline rs6219 & 12 & 102790192 & 0.04 & 0.11 & 3PRIME_UTR & $|G F|$ & 0.001688 & 2.97 & 0.3966 & 0.1272 & 1.39 \\
\hline rs2229616 & 18 & 58039276 & 0.04 & 0.01 & NON_SYNONYMOUS_CODING & $M C 4 R$ & 0.001784 & 0.24 & 0.4562 & 0.199 & 0.65 \\
\hline rs8141815 & 22 & 22191585 & 0.08 & 0.03 & INTRONIC & MAPKI & 0.001796 & 0.38 & 0.2854 & 0.2302 & 0.74 \\
\hline rs|38833| & 4 & 23778350 & 0.15 & 0.08 & INTERGENIC & PPARGCIA & 0.002473 & 0.50 & 0.6881 & 0.02169 & 0.67 \\
\hline rs2130925 & 4 & $2378 \mid 592$ & 0.09 & 0.04 & INTERGENIC & PPARGCIA & 0.00266 & 0.41 & 0.1013 & 0.00444 & 0.56 \\
\hline rs| 6859826 & 4 & 46992732 & 0.13 & 0.07 & INTRONIC & GABRA4 & 0.002676 & 0.49 & 0.7735 & 0.03276 & 0.68 \\
\hline rs 12595837 & 15 & 26870064 & 0.39 & 0.29 & INTRONIC & GABRB3 & 0.004517 & 0.64 & 0.5606 & 0.01708 & 0.75 \\
\hline rs 10886430 & 10 & 121010256 & 0.20 & 0.12 & INTRONIC & GRK5 & 0.005197 & 0.57 & 0.03149 & 0.0004877 & 0.58 \\
\hline rs|l| 40823 & 9 & 87614854 & 0.04 & 0.01 & INTRONIC & NTRK2 & 0.005407 & 0.28 & 0.7309 & 0.1419 & 0.59 \\
\hline rs I7292684 & 6 & 154554607 & 0.08 & 0.04 & INTRONIC & IPCEFI & 0.005623 & 0.43 & 0.4886 & 0.04363 & 0.65 \\
\hline rs I I085745 & 19 & 10824915 & 0.03 & 0.01 & INTRONIC & DNM2 & 0.005648 & 0.22 & 0.8015 & 0.0416 & 0.42 \\
\hline rs24274I2 & 20 & 61354135 & 0.09 & 0.16 & INTRONIC & NTSRI & 0.005668 & 2.01 & 0.09544 & 0.00264 & 1.73 \\
\hline rs9341077 & 6 & 152423128 & 0.07 & 0.03 & 3PRIME_UTR & ESRI & 0.005904 & 0.41 & 0.7668 & 0.04274 & 0.60 \\
\hline rs230050I & 14 & 90865815 & 0.10 & 0.05 & INTRONIC & CALMI & 0.005912 & 0.48 & 0.8348 & 0.1123 & 0.72 \\
\hline rs I03575I & 15 & 26943796 & 0.14 & 0.08 & INTRONIC & GABRB3 & 0.006047 & 0.53 & 0.2523 & 0.2147 & 0.79 \\
\hline rs|3|40445 & 4 & 46055623 & 0.42 & 0.32 & INTRONIC & GABRGI & $0.00628 \mid$ & 0.65 & 0.1703 & 0.004953 & 0.72 \\
\hline
\end{tabular}

Chr = chromosome; $M A$ freq $r e c=$ minor allele frequency in the recovered group; $M A$ freq ill = minor allele frequency in the ill group; disc GC $p$-value = genomic control-corrected $p$-values generated with the discovery cohort; disc OR=odds ratios generated with the discovery cohort; repl GC $p$-value $=$ genomic controlcorrected $p$-values generated with the replication cohort; comb GC p-value = genomic control-corrected $p$-values generated with the cohorts combined (ie, the raw data from all subjects); comb $O R=$ odds ratios generated with the combined cohort.

this association in our discovery cohort. No other SNPs showed a significant association by our criteria (ie, FDR $<0.05$ in the discovery sample, $p<0.05$ in the replication sample). We did, however, note that the second most strongly associated SNP from the analysis of the combined sample was rs4969170, which yielded an FDR $=0.0049$ $(\mathrm{OR}=0.55)$ and was located upstream of the gene SOCS3 on chromosome 17.

\section{Association of Flanking SNPs in LD with Rs17536211}

Utilizing the pairwise LD assessment function in PLINK, we obtained a measure of LD (ie, $R^{2}$ ) for rs17536211 vs all other SNPs in our data set. Supplementary Table 1 shows the 25 SNPs with the highest $R^{2}$ values and their corresponding $p$-value for association with ED outcome in our discovery cohort. In addition, Figure 1 provides a high-resolution Manhattan plot of this region and shows $p$-values for each genotyped SNP, which are color-coded as a function of $R^{2}$ value. As shown, in general, higher $R^{2}$ values correspond to lower $p$-values with respect to association with ED outcome, providing additional support for the validity of the original association with rs17536211.

\section{Haplotype Analyses}

Using a 3-SNP sliding window approach, haplotype analyses were conducted in our cohorts. Supplementary Table 2 shows the 25 most statistically significant 3-SNP haplotypes in our discovery cohort, as well as the corresponding $p$-values for each haplotype from our replication and combined samples, respectively. Of note, we report statistics from the omnibus test of association within a given window or locus. As shown, the top three haplotypes all include rs17536211, our top SNP from the single locus analysis. Results also suggest that rs17536211 alone is more strongly associated with ED outcome than any of the 3-SNP haplotypes we tested.

\section{Conditional and SNP $\times$ Rs17536211 Interaction Analyses}

Logistic regression analyses conditioning on rs17536211 did not reveal any other SNPs that were significantly associated with ED outcome. In the context of this analysis, however, the SNP for which we observed the strongest statistical evidence for association in our discovery cohort was rs2856966 (discovery, unadjusted $p=0.00034$; combined, unadjusted $p=0.00205)$, which is a non-synonymous coding SNP in ADCYAP1 on chromosome 18. In addition, 


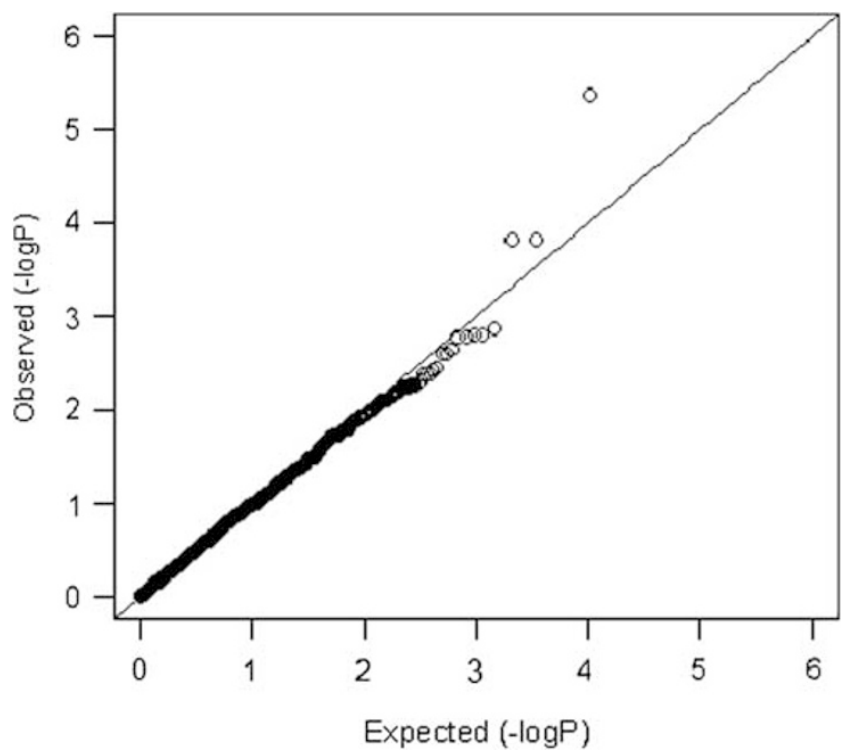

Figure I Q-Q plot for genetic association with eating disorder (ED) outcome in discovery sample.

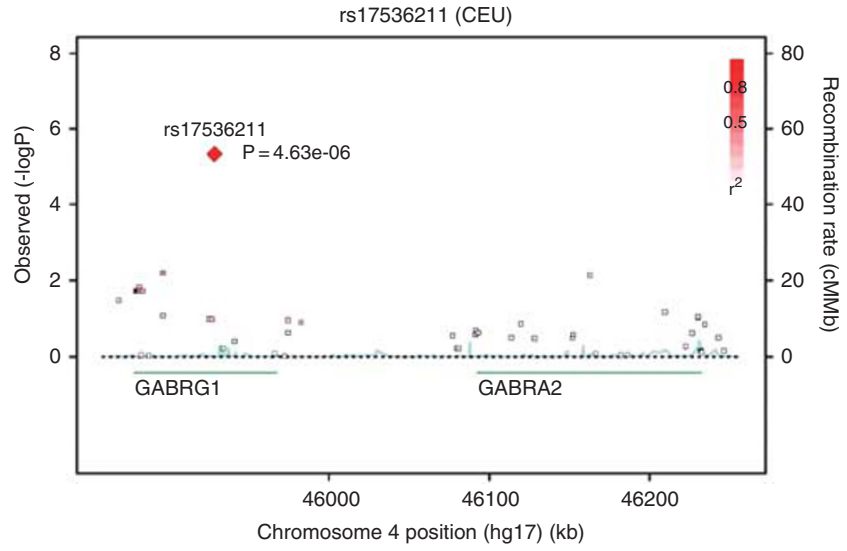

Figure 2 High-resolution Manhattan plot of the GABRGI and GABRA regions on chromosome 4. Plot shows $p$-values for each genotyped singlenucleotide polymorphism (SNP), which are color-coded as a function of $R^{2}$ value in relation to $r$ I $753621 \mathrm{I}$. As shown, higher $R^{2}$ values correspond to lower $p$-values with respect to association with eating disorder (ED) outcome, providing additional support for the validity of the original association with rs 1753621 I. Recombination break points are plotted in blue.

logistic regression analyses that included an SNP $\times$ rs17536211 interaction term were also conducted, but similarly, failed to find any SNPs that showed a significant interaction $p$-value. The SNP for which we observed the strongest statistical evidence for an interaction with rs17536211 in our discovery cohort was rs30297 (discovery, unadjusted interaction $p=2.67 \times 10^{-5}$; combined, unadjusted interaction $p=0.011$ ), which is an intergenic SNP near ADRB2 on chromosome 5.

\section{Evaluation of GABA SNPs in the Data Set as a Whole}

Our most strongly associated SNP was a GABA receptor SNP, and 10 out of the 25 most strongly associated haplotypes involved SNPs in GABA genes. Therefore, we attempted to more formally evaluate the extent to which GABA SNPs were over-represented among the most strongly associated SNPs from our single locus analyses. The results of this analysis are presented in Table 3. As shown, $7.4 \%$ of GABA receptor SNPs $v s$ only $4.2 \%$ of non-GABA SNPs show $p$-values of $<0.05$ in analysis of the discovery cohort $(Z=3.64, p=0.0003)$. Although this finding does not hold up in the replication cohort alone, we did observe the same pattern, although somewhat attenuated, in the combined sample $(Z=2.07, p=0.0388)$, suggesting over-representation of GABA SNPs among SNPs most strongly associated with ED outcome.

\section{Follow-Up Phenomic Association Analyses}

In a third independent cohort of women, we conducted phenomic association analyses of rs17536211 with age at menarche, anxiety, and perfectionism (Table 4). As illustrated, rs 17536211 shows a nominally significant association with trait anxiety $(p=0.049)$. We note that a Bonferroni adjustment to this analysis of three traits, this result would not survive correction; thus, we repeated this analysis for all of the individuals in the data set (ie, all 1878) and found consistent results with this larger sample (rs17536211 associated with trait anxiety at $p=0.036$ ). Further exploration of the direction of the effect also revealed that individuals with two copies of the minor allele showed lower levels of trait anxiety (Figure 3 ) relative to major allele carriers, which is consistent with the observation that this variant exhibits protective effects. Neither perfectionism (ie, Concern Over Mistakes) nor age at menarche was found to be associated with rs 17536211 .

\section{DISCUSSION}

Within the context of a large-scale candidate gene study originally designed to evaluate candidate genes that may have a role in the genetic susceptibility to EDs (Pinheiro et al, 2010), we tested for genetic association with recovery from EDs leveraging a total sample size of 1878 women. Utilizing a tiered design in which we first performed these analyses in a strictly defined discovery cohort and then in a more liberally defined replication cohort, we identified evidence of association for an intronic SNP (rs17536211) in GABRG1 on chromosome 4. LD patterns and association results for flanking SNPs provided additional support for the validity of this association. Follow-up phenomic association of our top SNP revealed a nominal association with trait anxiety, suggesting a possible mechanism through which this variant may influence ED outcome. Taken together, these findings may provide new insights into mechanisms related to poor outcome, and thus aid in the development of more effective interventions for the most treatment-resistant patients.

\section{Overview of GABA-A Receptors}

GABRG1, which codes the $\gamma$-1-subunit, belongs to the ligand-gated ionic channel family and combines with other subunits to form GABA-A receptors (Michels and Moss, 2007). GABA is the major inhibitory neurotransmitter in the central nervous system. The inhibitory effect of GABA is 
Table 3 Evaluation of GABA SNPs in the Data Set as a Whole

\begin{tabular}{|c|c|c|c|c|c|}
\hline Chr. & Gene & SNPs in data set & $\begin{array}{l}\text { SNPs } p<0.05 \text { in } \\
\text { discovery sample }\end{array}$ & $\begin{array}{l}\text { SNPs } p<0.05 \text { in } \\
\text { replication sample }\end{array}$ & $\begin{array}{l}\text { SNPs } p<0.05 \text { in } \\
\text { combined sample }\end{array}$ \\
\hline 4 & GABRA2 & 25 & I (4\%) & $0(0 \%)$ & I (4\%) \\
\hline 4 & GABRB I & 76 & I (I\%) & $9(12 \%)$ & I (I\%) \\
\hline 4 & GABRG I & 23 & I I (48\%) & I I (48\%) & $13(56 \%)$ \\
\hline 5 & GABRB2 & 50 & $3(6 \%)$ & $5(10 \%)$ & $2(4 \%)$ \\
\hline 5 & GABRG2 & 33 & $0(0 \%)$ & I (3\%) & $0(0 \%)$ \\
\hline 5 & GABRP & 25 & $0(0 \%)$ & $0(0 \%)$ & $0(0 \%)$ \\
\hline 6 & GABBRI & 16 & $0(0 \%)$ & $6(38 \%)$ & $4(25 \%)$ \\
\hline 6 & GABRRI & 29 & $5(17 \%)$ & $0(0 \%)$ & $0(0 \%)$ \\
\hline 15 & GABRG3 & 132 & $13(10 \%)$ & $7(5 \%)$ & $3(2 \%)$ \\
\hline 16 & GABARAPL2 & 4 & I (25\%) & $0(0 \%)$ & I (25\%) \\
\hline$x$ & GABRA3 & 46 & $0(0 \%)$ & $0(0 \%)$ & $0(0 \%)$ \\
\hline$x$ & GABRE & 4 & $0(0 \%)$ & $0(0 \%)$ & $0(0 \%)$ \\
\hline \multirow[t]{4}{*}{$X$} & GABRQ & 2 & $0(0 \%)$ & $0(0 \%)$ & $0(0 \%)$ \\
\hline & Total in GABA genes & 652 & $48(7.4 \%)$ & $43(6.6 \%)$ & $36(5.5 \%)$ \\
\hline & Total in non-GABA genes & 4499 & $190(4.2 \%)$ & $279(6.2 \%)$ & 169 (3.8\%) \\
\hline & Total & $5|5|$ & $238(4.6 \%)$ & $322(6.2 \%)$ & $205(4.0 \%)$ \\
\hline
\end{tabular}

Bold value indicates $p<0.05$, which is the statistical significance threshold.

Table 4 Follow-up Phenomic Association Analyses

\begin{tabular}{lccccccc}
\hline Dependent variable & $\boldsymbol{\beta} \mathbf{I}(\mathbf{D X})$ & $\mathbf{D X} \boldsymbol{p}$-value & $\boldsymbol{\beta 2} \mathbf{( B M I )}$ & $\mathbf{B M I} \boldsymbol{p}$-value & $\boldsymbol{\beta 3}$ (rs I 75362II) & SNP $\boldsymbol{p}$-value & $\boldsymbol{R}^{\mathbf{2}}$ change with rs I75362I I \\
\hline STAl trait anxiety & 0.682 & $<0.0005$ & -0.085 & 0.002 & -0.048 & $\mathbf{0 . 0 4 9 *}$ & 0.002 \\
Concern over mistakes & 0.663 & $<0.0005$ & -0.032 & 0.270 & 0.008 & 0.753 & $<0.0005$ \\
Age at menarche & 0.057 & 0.138 & -0.185 & $<0.0005$ & -0.037 & 0.284 & 0.001 \\
\hline
\end{tabular}

$\beta I=$ regression coefficient associated with the effect of diagnosis; DX $p$-value $=p$-value associated with the effect of diagnosis

$\beta 2=$ regression coefficient associated with the effect of body mass index; BMI $p$-value $=p$-value associated with the effect of body mass index

$\beta 3=$ regression coefficient associated with the effect of 'top' SNP; SNP $p$-value = $p$-value associated with the effect of 'top' SNP

*Indicates statistically significant at $p<0.05$.

mediated either by GABA-A receptors, which are ionotropic GABA-gated chloride channel receptors, or by the metabotropic GABA-B receptors. GABA-A receptors are heteromeric pentamers composed of five subunits that can belong to different subfamilies (Michels and Moss, 2007). To date, 19 different subunits have been isolated, including $\alpha 1-6, \beta 1-3, \gamma 1-3, \delta, \varepsilon, \pi, \rho 1-3$, and $\theta$. Of the 19 GABA genes included in our data set, 16 were GABA-A receptor subunit genes (see Table 3). Receptors containing the $\alpha 1-, \alpha 2-, \alpha 3-$, or $\alpha 5$-subunit in combination with any of the $\beta$-subunits and the $\gamma 2$-subunits are most prevalent in the brain.

\section{GABRG1 and the $\gamma 1$-Subunit}

For rs17536211, an intronic SNP in GABRG1, possession of copies of the minor allele was protective from long-term chronic illness (ie, associated with recovery). Moreover, individuals with two copies of the rs17536211 GABRG1 minor allele showed lower levels of trait anxiety relative to major allele carriers, which is consistent with the possibility that the minor allele variant exhibits protective effects. Consistent with the idea that more extreme, developmentally antecedent anxiety phenotypes may confer risk of greater illness morbidity is evidence that individuals who remain chronically ill have significantly higher levels of anxiety compared with those that recover (Kaye et al, 2004a), and that childhood anxiety is associated with an illness trajectory marked by an overall greater degree of caloric restriction and ED psychopathology, as well as a lower lifetime BMI (Dellava et al, 2010; Raney et al, 2008). Although evidence from Kaye et al (2004a, b) did not establish whether increased anxiety in those that are chronically 


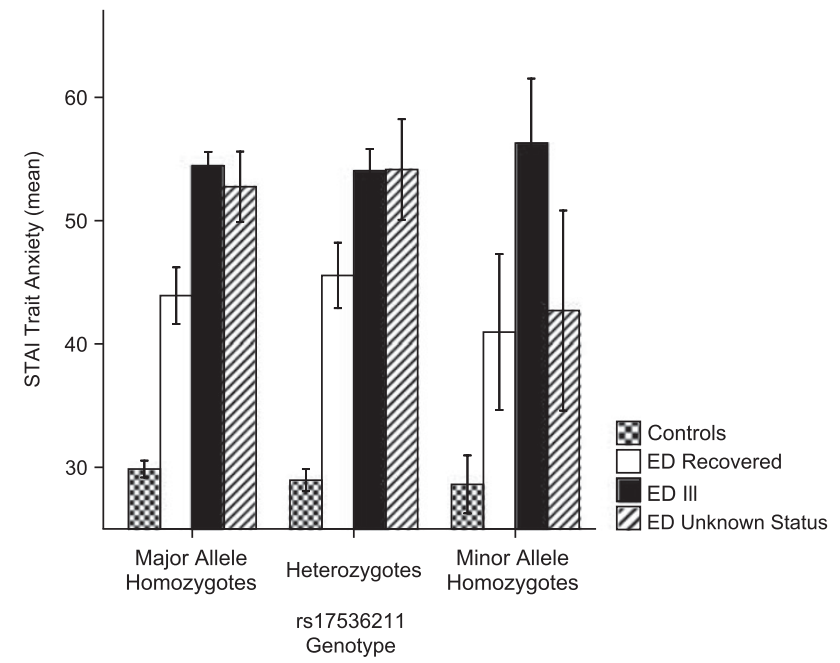

Figure 3 Trait anxiety as a function of diagnosis, recovery status, and rs I75362 I I genotype. As shown, all study groups with the exception of the ill group show lower trait anxiety among minor allele homozygotes, consistent with the statistically significant association between trait anxiety and rs 1753621 I genotype. Error bars represent the 95\% confidence interval.

ill is an antecedent or consequence of malnutrition, the studies by Dellava et al (2010) and Raney et al (2008) cited above established the precedence of anxiety onset in relationship to later ED psychopathology, and thus converge with findings in this study that genetic predisposition to more extreme anxiety may predispose some with AN to a more intractable disease.

It has been noted (Covault et al, 2008) that the expression of the $\gamma 1$-subunit, in comparison to most GABA-A subunits, is limited to very few brain areas, including the pallidum, septum, bed nucleus of the stria terminalis, and the central and medial amygdaloid nuclei (Akari et al, 1992; Persohn et al, 1992; Pirker et al, 2000; Wisden et al, 1992; Ymer et al, 1990). The functional influence, if any, of the rs17536211 SNP in human beings remains to be discovered. Still, limited data implicate the $\gamma 1$-subunit in modulation of anxiety. Esmaeili et al (2009) found that GABA-A receptor subunits were differentially distributed in the amygdala using pharmacological profiles generated from in vitro expression studies in HEK293 cells. The basolateral and central amygdala GABA-A receptors contained $\alpha 2-, \beta$-, and $\gamma 2$-subunit. In contrast, GABA receptors containing $\gamma 1$-subunits were found in the lateral inputs, a region that arises from the intercalated cells masses, and is thought to be responsible for mediating inhibition of amygdala output during extinction of conditioned fear (Likhtik et al, 2008). Interestingly, there has been speculation that individuals with AN have altered extinction (Petrovich et al, 2009; Strober 2004), and much evidence shows that brain regions containing the $\gamma 1$ GABA-A subunit are substrates for the regulation of anxiety states and various aspects of appetitive motivation (Berridge, 2009; LeDoux, 2000), suggesting that exploration of the relationship of clinical phenomena to $\gamma 1$-subunit containing GABA-A receptor function may be worthwhile.

\section{Implication for Treatments of Poor Outcome}

$\mathrm{AN}$ and $\mathrm{BN}$ are deadly disorders, with a lifetime mortality rate of $>5 \%$. Those with poor outcome are at the greatest risk. Findings from this study hold the promise of shedding light on new targets for the treatment of those with poor outcome. It is well known that benzodiazepines produce their potent anxiolytic therapeutic actions on GABA-A receptors (Esmaeili et al, 2009; Rudolph and Mohler, 2006). Interestingly, the GABRG1 gene confers effects on GABA-A receptors that alter the response to benzodiazepines. That is, $\gamma 1$-containing GABA-A receptors show reduced sensitivity to benzodiazepine agents (Covault et al, 2008; Esmaeili et al, 2009; Khom et al, 2006; Ymer et al, 1990). To our knowledge, there is little in the way of trials of any GABA-related drug in EDs. Our clinical experience is that benzodiazepines are not effective treatments for anxiety in individuals with AN. Whether drugs that interact with $\gamma 1$-containing GABA-A receptors might show improved efficacy in those with poor outcome remains to be discovered.

\section{GABRG1 and Alcoholism}

GABRG1 has also been associated with the level of response to alcohol, as well as drinking behaviors (Covault et al, 2008; Enoch et al, 2009; Ray and Hutchinson, 2009). These studies suggest that an association signal between alcoholuse disorders (AUDs) and GABRA2 might have arisen from a functional variant in the neighboring GABRG1 gene. Two studies (Covault et al, 2008; Enoch et al, 2009), each with two populations, showed replication of haplotype and SNP associations with alcoholism in a haplotype block that extends from GABRG1 intron 2 to the intergenic region between GABRG1 and GABRA2. However, the family-based Collaborative Studies on Genetics of Alcoholism data set did not show association of GABRG1 SNPs extending from the $5^{\prime}$ region to intron 8 (Edenberg et al, 2004).

Of the 31 GABRG1 SNPs previously genotyped for AUDs, eight SNPs were genotyped in this ED study. A comparison of the seven SNPs is common between the Enoch study (Enoch et al, 2009) and our data are given in Table 5. These SNPs showed comparable minor allele frequency differences between alcoholics $v s$ controls and ED ill $v s$ ED recovered. Still, identification of minor and major allele frequencies between studies can be problematic. Specifically, these studies involve two different populations where a minor allele in one population could be a major allele in the other population and vice versa. Also, owing to potential strand differences between the two studies, precise allelic comparisons can be difficult, and we cannot be entirely certain as to whether the same allele is implicated in ED outcome and alcoholism. Conservatively, although it is not clear whether chronic illness in ED and alcoholism have similar or opposite susceptibility factors, it can be concluded that there is an overlap of implicated SNPs in both studies.

There may be several explanations for this concordance. First, there may be occult population substructure in the GABRG1 gene region, explaining the case-control differences in both alcoholism and EDs. However, this population substructure hypothesis would not address the similar findings in several other GABA genes located in other areas 
Table 5 GABRGI SNP Minor Allele Frequency Differences in ED III vs Recovered (This Study) and Alcohol-Use Disorders vs Control (Enoch et al, 2009)

\begin{tabular}{|c|c|c|c|c|c|c|c|c|c|c|c|}
\hline \multirow{2}{*}{ SNP } & \multirow{2}{*}{ Type } & \multirow{2}{*}{ Minor } & \multirow{2}{*}{ Major } & \multicolumn{2}{|c|}{ Eating disorder } & \multicolumn{2}{|c|}{ Alcoholism } & \multirow{2}{*}{$\begin{array}{c}\text { ED } \\
p \text {-value }\end{array}$} & \multirow{2}{*}{$\begin{array}{l}\text { ETOH } \\
p \text {-value }\end{array}$} & \multicolumn{2}{|c|}{ MAF frequency difference } \\
\hline & & & & MAF ill & MAF rec & MAF alc & MAF ctrl & & & ED ill vs rec & ETOH alc vs ctrl \\
\hline rs| 497568 & 3PRIME_UTR & G & A & 0.45 & 0.54 & 0.48 & 0.56 & 0.01901 & 0.02 & -0.0892 & -0.08 \\
\hline rs|753621। & INTRONIC & C & $A$ & 0.18 & 0.33 & 0.19 & 0.24 & 4.63E-06 & 0.137 & -0.144 & -0.05 \\
\hline rs|7536530 & INTERGENIC & C & A & 0.08 & 0.06 & 0.11 & 0.07 & 0.2339 & 0.079 & 0.02381 & 0.04 \\
\hline rs|7639557 & INTERGENIC & $\mathrm{T}$ & C & 0.46 & 0.53 & 0.41 & 0.5 & 0.1105 & 0.019 & -0.0607 & -0.09 \\
\hline rs6447493 & 3PRIME_UTR & $\mathrm{T}$ & C & 0.45 & 0.54 & 0.48 & 0.56 & 0.01865 & 0.012 & -0.09 & -0.08 \\
\hline rs976156 & SYNONYMOUS_CODING & $\mathrm{T}$ & C & 0.45 & 0.39 & 0.42 & 0.35 & 0.1046 & 0.03 & 0.0613 & 0.07 \\
\hline rs993677 & INTRONIC & C & $\mathrm{T}$ & 0.12 & 0.08 & 0.12 & 0.11 & 0.08544 & 0.478 & 0.04084 & 0.01 \\
\hline
\end{tabular}

$\mathrm{MAF}=$ minor allele frequency.

$p$-value $=p$-value observed in this study comparing women ill vs recovered from an eating disorder; ETOH $p$-value $=p$-value observed in the Enoch et al (2009) study comparing individuals with alcohol-use disorders vs controls.

of the genome. Second, the GABRG1 SNP allele frequency differences may be attributable to a characteristic that the individuals with alcoholism share with the persistently ill ED probands, such as compulsive behaviors. That is, individuals with alcoholism drink compulsively, whereas ill ED probands engage in persistent, compulsive food refusal and exercise. Third, the GABRG1 SNP allele frequency differences may be attributable to an anxious temperament, a characteristic that is found to a greater degree in persistently ill ED probands (compared with the recovered ED probands) and in individuals with alcoholism compared with controls. Thus, the possibility is raised that the associated allele might reflect a form of anxiety that may be shared by persistently ill ED probands and many individuals with alcoholism. Furthermore, research has shown an association between EDs and substance-use disorders, including alcohol use (Baker et al, 2010), although AUDs are significantly more prevalent in women with ANBN and $\mathrm{BN}$ than in women with AN (Bulik et al, 2004).

\section{Findings in Other GABA Subunits}

GABA SNPs were found to be over-represented among SNPs associated with ED outcome in the data set as a whole. This suggests that a complex genetic pattern of GABAergic transmission may predispose to a chronic course of ED illness, but this finding must be confirmed in independent cohorts. Before such confirmation is reported, it is prematurely speculative to consider a more detailed hypothesis.

\section{Limitations}

This study has several strengths, including a relatively large total sample size, detailed and in-depth phenotypic characterization of participants, selection of high probability candidate genes and coding SNPs, and application of conservative QC procedures and widely accepted multiple test correction procedures. We do, however, note some limitations, including smaller samples of individuals in the ED 'recovery' groups, retrospective data collection susceptible to various recall biases, and perhaps a less stringent definition of recovery in terms of timeframe (ie, no ED symptoms within the past year) than has been used in some previous studies of outcome (Von Holle et al, 2008). In addition, the relatively low recovery rates observed in this study (ie, much $<50 \%$ ) raises the possibility that our cases, ascertained for a genetic study, differ from clinical or community samples in terms of ED pathology, co-morbidity, and course of illness. Further, we have assumed a single recovery event, and also do not account for women who may have died from an ED. We also note that although this was a candidate gene study, if genome-wide statistical significance criteria were applied to the interpretation of results (eg, $p<5 \times 10^{-7}$ ), our top hit would not have reached statistical significance.

\section{ACKNOWLEDGEMENTS}

We thank the Price Foundation for the support of clinical data and biospecimen collection, genotyping, and data analysis. We further acknowledge the participating families and patients without whom this work would not have been possible. This work was supported by the Price Foundation of Geneva, Switzerland. Drs Bloss and Schork are additionally supported, in part, by an NIH/NCRR flagship Clinical and Translational Science Award Grant (1U54RR025204-01).

\section{DISCLOSURE}

Dr Kaye has received salary support from the University of Pittsburgh and the University of California, San Diego; research funding/support from the NIMH; research funding for an investigator-initiated treatment study from AstraZeneca and consulting fees from Lundbeck, Merck, and the Eating Disorder Center of Denver. In addition, there are honoraria for presentations from academic institutions and meetings, and compensation for grant review activities from the National Institutes of Health. Dr Crow has received support in the past 3 years from Eli Lilly, Pfizer, lVJovartis, and GSK. Dr Bergen has received compensation from the Center for Scientific Review, National Institutes of Health, and from the National Coalition for Health Professional 
Education in Genetics. The remaining authors, CS Bloss, C Johnson, DB Woodside, S Crawford, H Brandt, A Kaplan, M Strober, P Magistretti, K Halmi, E Marzola, K Klump, J Treasure, M Fichter, J Mitchell, P Keep, W Berrettini, V Duvvuri, and NJ Schork declare that, except for income received from our primary employers and the abovementioned funding, no further financial support or compensation has been received from any individual or corporate entity over the past 3 years for research or professional service and there are no personal financial holdings that could be perceived as constituting a potential conflict of interest.

\section{REFERENCES}

Akari T, Kiyama H, Tohyama M (1992). The GABAA receptor gamma 1 subunit is expressed by distinct neuronal populations. Brain Res 15: 121-132.

American Psychiatric Association (2000). Diagnostic and Statistical Manual of Mental Disorders - Fourth Edition - Text Revision (DSM-IV-TR). American Psychiatric Association.

Baker J, Mitchell K, Neale MC, Kendler KS (2010). Eating disorder symptomatology and substance use disorders: prevalence and shared risk in a population based twin sample. Int J Eat Disord 43: 846-658.

Benjamini Y, Drai D, Elmer G, Kafkafi N, Golani I (2001). Controlling the false discovery rate in behavior genetics research. Behav Brain Res 125: 279-284.

Benjamini Y, Hochberg Y (1995). Controlling the false discovery rate: a practical and powerful approach to multiple testing. $J$ R Statist Soc Ser B 57: 289-300.

Berridge K (2009). 'Liking' and 'wanting' food rewards: brain substrates and roles in eating disorders. Physiol Behav 97: 537-550.

Bulik C, Bacanu S, Klump K, Fichter M, Halmi K, Keel P et al (2005). Selection of eating-disorder phenotypes for linkage analysis. Am J Med Genet B 139: 81-87.

Bulik C, Berkman N, Brownley K, Sedway J, Lohr K (2007). Anorexia nervosa treatment: a systematic review of randomized controlled trials. Int J Eat Disord 40: 310-320.

Bulik C, Klump K, Thornton L, Kaplan A, Devlin B, Fichter M et al (2004). Alcohol use disorder comorbidity in eating disorders: a multicenter study. J Clin Psychiatry 65: 1000-1006.

Bulik C, Tozzi F, Anderson C, Mazzeo S, Aggen S, Sullivan P (2003). The relation between eating disorders and components of perfectionism. Am J Psychiatry 160: 366-368.

Covault J, Gelernter J, Jensen K, Anton R, Franzier H (2008). Markers in the $5^{\prime}$-region of GABRG1 associate to alcohol dependence and are in linkage disequilibrium with markers in the adjacent GABRA2 gene. Neuropsychopharmacology 33: 837-848.

Crow S, Petersen C, Swanson S, Raymond N, Specker S, Eckert E (2009). Increased mortality in bulimia nervosa and other eating disorders. Am J Psychiatry 166: 1342-1346.

Dellava J, Thornton LH, Hamer R, Strober M, Plotnicov K, Klump K et al (2010). Childhood anxiety associated with low BMI in women with anorexia nervosa. Behav Res Ther 48: 60-67.

Devlin B, Bacanu S-A, Klump KL, Bulik CM, Fichter MM, Halmi KA et al (2002). Linkage analysis of anorexia nervosa incorporating behavioral covariates. Hum Mol Genet 11: 689-696.

Devlin B, Roeder K (1999). Genomic control for association studies. Biometrics 55: 997-1004.

Edenberg H, Dick D, Xuei X, Tian H, Almasy L, Bauer L et al (2004). Variations of GABRA2, encoding the alpha 2 subunit of the GABA(A) receptor, are associated with alcohol dependence and with brain oscillations. Am J Hum Genet 74: 705-714.
Enoch M, Hodgkinson C, Yuan Q, Albaugh B, Birkkunen M, Goldman D (2009). GABRG1 and GABRA2 as independent predictors for alcoholism in two populations. Neuropsychopharmacology 34: 1245-1254.

Esmaeili A, Lynch JW, Sah P (2009). GABAA receptors containing gamma 1 subunits contribute to inhibitory transmission in the central amygdala. J Neurophysiol 101: 341-349.

First M, Spitzer R, Gibbon M, Wiloliams J (1997). Structured Clinical Interview for DSM-IV Axis I Disorders, Research Version, Patient Edition. Biometrics Research, New York State Psypchiatric Institute: New York.

Frost RO, Marten P, Lahart C, Rosenblate R (1990). The dimensions of perfectionism. Cogn Ther Res 14: 449-468.

Ge D, Zhang K, Need A, Martin O, fellay J, urbasn T et al (2008). WGAViewer: software for genomic annotation of whole genome association studies. Genome Res 18: 640-643.

Grice DE, Halmi KA, Fichter M, Strober M, Woodside DB, Treasure $\mathrm{J}$ et al (2002). Evidence for a susceptibility gene for anorexia nervosa on chromosome 1. Am J Hum Genet 70: 787-792.

Hemminger B, Saelim B, Sullivan P (2006). TAMAL: an integrated approach to choosing SNPs for genetic studies of human complex traits. Bioinformatics 22: 626-627.

Kaye W, Bulik C, Thornton L, Barbarich N, Masters K, Fichter M et al (2004a). Comorbidity of anxiety disorders with anorexia and bulimia nervosa. Am J Psychiatry 161: 2215-2221.

Kaye WH, Devlin B, Barbarich N, Bulik C, Thornton L, Bacanu SA et al (2004b). Genetic analysis of bulimia nervosa: methods and sample description. Int J Eat Disord 35: 556-570.

Kaye W, Fudge J, Paulus M (2009). New insight into symptoms and neurocircuit function of anorexia nervosa. Nat Rev Neurosci 10: 573-584.

Kaye WH, Lilenfeld LR, Berrettini WH, Strober M, Devlin B, Klump KL et al (2000). A search for susceptibility loci for anorexia nervosa: methods and sample description. Biol Psychiatry 47: 794-803.

Keel P, Brown T (2010). Update on course and outcome in eating disorders. Int J Eat Disord 43: 195-204.

Keel PK, Mitchell JE, Miller KB, Davis TL, Crow SJ (1999). Long-term outcome of bulimia nervosa. Arch Gen Psychiatry 56: 63-69.

Khom S, Baburin I, Timin EN, Hohaus A, Sieghart W, Hering S (2006). Pharmacological properties of GABAA receptors containing gammal subunits. Mol Pharmacol 69: 640-649.

LeDoux J (2000). Emotion circuits in the brain. Ann Rev Neurosci 23: $155-164$.

Likhtik E, Popa D, Apergis-Schoute J, Fidacaro G, Pare D (2008). Amygdala intercalated neurons are required for expression of fear extinction. Nature 454: 642-645.

Lilenfeld L, Wonderlich S, Riso LP, Crosby R, Mitchell J (2006). Eating disorders and personality: a methodological and empirical review. Clin Psychol Rev 26: 299-320.

Lilenfeld LR, Kaye WH, Greeno CG, Merikangas KR, Plotnicov K, Pollice C et al (1998). A controlled family study of anorexia nervosa and bulimia nervosa: psychiatric disorders in firstdegree relatives and effects of proband comorbidity. Arch Gen Psychiatry 55: 603-610.

Michels G, Moss S (2007). GABAA receptors: properties and trafficking. Crit Rev Biochem Mol Biol 42: 3-14.

Persohn E, Malherbe P, Richards J (1992). Comparative molecular neuroanatomy of closed BAGAA receptor subunits in the rat CNS. J Comp Neurol 326: 193-216.

Petrovich G, Ross CA, Mody P, Holland PC, Gallagher M (2009). Central, but not basolateral, amygdala is critical for control of feeding by aversive learned cues. J Neurosci 29: 15205-15212.

Pinheiro A, Bulik C, Thornton L, Sullivan P, Root T, Bloss C et al (2010). Association study of 182 candidate genes in anorexia nervosa. Am J Med Genet B 153B: 1070-1080. 
Pirker S, Wieselthaler A, Sieghart W, Sperk G (2000). GABA(A) receptor: immunocytochemical distribution of 13 subunits in the adult rat brain. Neurosci 101: 815-850.

Pruitt K, Tatusova T, Maglott D (2007). NCBI reference sequences (RefSeq): a curated non-redundant sequence database of genomes, transcripts and proteins. Nucleic Acids Res 35: D61-D65.

Purcell S, Neale B, Todd-Brown K, Thomas L, Ferreira M, Bender D (2007). PLINK: a tool set for whole-genome association and population-based linkage analyses. Am J Hum Genet 81: 559-575.

Raney T, Thornton L, Brandt H, Crawford S, Fichter M, Halmi K et al (2008). Influence of overanxious disorder of childhood on the expression of anorexia nervosa. Int J Eat Disord 41: 326-332.

Ray L, Hutchinson K (2009). Associations among GABRG1, level of response to alcohol, and drinking behaviors. Alcohol Clin Exp Res 33: 1382-1390.

Reba L, Thornton L, Tozzi F, Klump K, Brandt H, Crawford S et al (2005). Relationship between features associated with vomiting in purging-type eating disorders. Int J Eat Disord 38: 287-294.

Rudolph U, Mohler H (2006). GABA-based therapeutic approaches: GABAA receptor subtype functions. Cur Opin Pharmacol 6: 18-23.

Shapiro J, Berkman N, Brownley K, Sedway J, Lohr K, Bulik C (2007). Bulimia nervosa treatment: a systematic review of randomized controlled trials. Int J Eat Disord 40: 321-336.

Spielberger C, Luschene P, Vagg P, Jacobs A (1983). Manual for the State-Trait Anxiety Inventory (Form Y). Consulting Psychologists Press: Palo Alto, CA.

Steinhausen H (2009). Outcome of eating disorders. Child Adolesc Psychiatric Clin N Am 18: 225-242.

Steinhausen H, Weber S (2009). The outcome of bulimia nervosa: findings from one-quarter century of research. Am J Psychiatry 166: $1331-1341$.
Steinhausen HC (2002). The outcome of anorexia nervosa in the 20th century. Am J Psychiatry 159: 1284-1293.

Strober M (2004). Pathologic fear conditioning and anorexia nervosa: on the search for novel paradigms. Int $J$ Eat Disord 35: 504-508.

Sullivan PF (1995). Mortality in anorexia nervosa. Am J Psychiatry 152: 1073-1074.

Versini A, Ramoz N, Le Strat Y, Scherag S, Ehrlich S, Boni C et al (2010). Estrogen receptor 1 gene (ESR1) is associated with restrictive anorexia nervosa. Neuropsychopharmacology 35: 18-25.

Von Holle A, Pinheiro A, Thornton L, Klump K, Berrettini W, Brandt $\mathrm{H}$ et al (2008). Temporal patterns of recovery across eating disorder subtypes. Aust N Z J Psychiatry 42: 108-117.

Wagner A, Barbarich N, Frank G, Bailer U, Weissfeld L, Henry S et al (2006a). Personality traits after recovery from eating disorders: do subtypes differ? Int J Eat Disord 39: 276-284.

Wagner A, Greer P, Bailer U, Frank G, Henry S, Putnam K et al (2006b). Normal brain tissue volumes after long-term recovery in anorexia and bulimia nervosa. Biol Psychiatry 59: 291-293.

Walsh B (1991). A parent's guide to anorexia and bulimia. Understanding and helping self-starvers and binge/purgers. J Child Adolesc Psychopharmacol 1: 388-389.

Walters EE, Kendler KS (1995). Anorexia nervosa and anorexiclike syndromes in a population-based female twin sample. Am J Psychiatry 152: 64-71.

Wisden W, Laurie D, Monyer H, Seeburg P (1992). The distribution of 13 GABAA receptor subunit mRNAs in the rat brain. I. Telencephalon, diencephalon, mesencephalon. J Neuroscience 12: 1040-1062.

Ymer S, Draguhn A, Wisden W, Werner P, Keinanen K, Schofield P et al (1990). Structural and functional characterization of the gamma 1 subunit of GABAA/benzodiazepine receptors. EMBO J 9: 3261-3267.

Supplementary Information accompanies the paper on the Neuropsychopharmacology website (http://www.nature.com/npp) 